\title{
STABILITY OF AN SIRS EPIDEMIC MODEL WITH A VARIABLE INCIDENCE RATE AND TIME DELAY
}

\author{
YOUNG IL SEO ${ }^{1}$, GI PHIL CHO ${ }^{2}$, KYOUNG SOOK CHAE $^{2}$, AND IL HYO JUNG ${ }^{2}$ \\ ${ }^{1}$ National Fisheries Research and Development Institute, Busan 619-705, South Korea \\ E-mail address: seovi@nnfrdi.go.kr \\ 2 Department of Mathematics, Pusan National University, Pusan 609-735, South Korea \\ E-mail address: yogofo@naver.com, shiral@nate.com, ilhjung@pusan.ac.kr
}

\begin{abstract}
The purpose of this paper is to prove existence of solutions of an $S I R S$ epidemic model with time delay of continuous type and the variable incidence rate and to investigate some asymptotic behaviors of the SIRS epidemic model. An example illustrating the stability of the model is given. The results extend the corresponding results in the literature.
\end{abstract}

\section{INTRODUCTION}

Mathematical epidemiology seems to have grown largely starting in the middle of the 20th century $[1,3]$ and a tremendous variety of models have now been formulated, mathematically analyzed, and applied to infectious diseases. These models are defined in mathematical modeling forms with respect to disease status and consist generally of three components: susceptible $(S)$ individuals that have been noninfected, and thus they are able to catch the disease, infected $(I)$ individuals who are infected can spread the disease to susceptible individuals. The time that individuals spend in the infected state is called the infectious period; after they enter the recovered state, and recovered $(R)$ individuals in the recovered state are assumed to be immune for life.

Several epidemic models and reviews on theoretical developments with stability analysis are described in $[4,5,7,10]$. Recently, much attention has been given to the persistence and global stability of the epidemic model with time delay.

Received by the editors August 25 2012; Accepted March 62013.

1991 Mathematics Subject Classification. 92D30, 34D20.

Key words and phrases. Existence, Epidemic model, Stability, Endemic equilibrium state, Lyapunov function.

This work was supported by Basic Science Research Program through the National Research Foundation of Korea (NRF) funded by the Ministry of Education, Science and Technology (MEST) (2012-000599). 
In 2004, Ma et al.[6] studied the global asymptotic stability of the $S I R$ epidemic model with time delay given by

$$
\begin{aligned}
& \frac{d S}{d t}=\Lambda-\beta S(t) I(t-\tau)-\mu_{1} S(t), \\
& \frac{d I}{d t}=\beta S(t) I(t-\tau)-\left(\mu_{2}+\gamma\right) I(t), \\
& \frac{d R}{d t}=\gamma I(t)-\mu_{3} R(t) .
\end{aligned}
$$

Here the positive constant $\Lambda$ is the immigration rate, assumed all newborns to be susceptible, $\gamma$ is the recovery rate of infected individuals, and $\mu_{1} \geq 0, \mu_{2} \geq 0$ and $\mu_{3} \geq 0$ represent the death rates of the susceptible, infected and recovered, respectively. Also the constant $\tau \geq 0$ is the time delay.

Most of previous models assumed that an incidence rate is constant and a delay term is discrete type about an infected term and so these models may have the limits of realistic descriptions. In order to make a more realistic situation, we propose a continuous delay model with measure $d \eta(s)$ about the delay which is in the infected term $I$. The function $\eta(s):[0, \tau] \rightarrow(-\infty, \infty)$ is nondecreasing and has $\int_{0}^{\tau} d \eta(s)=\eta(\tau)-\eta(0)=A>0$. Moreover, we propose a nonconstant function $\beta(I)$ which is an incidence rate per unit time. Thus, $\beta(I) S(t) \int_{0}^{\tau} I(t-s) d \eta(s)$ means the number of new infections in unit time. We assume that $\beta(I)$ is a positive continuous function and there exists a constant $\tilde{I}>0$ such that $\beta(I)$ is nondecreasing on the interval $[0, \tilde{I}]$. Also, this epidemic model may have recovered individuals with temporary immunity, that is, the loss of immunity by immune individuals allows the disease to become endemic. So we have the relation $S \rightarrow I \rightarrow R \rightarrow S$. Note that the total individuals $N(t)=S(t)+I(t)+R(t)$ may be change in time $t$. A summary of the process is drawn in a flow chart in Figure 1.

In this paper, we consider an $S I R S$ epidemic model with time delay of continuous type as follow:

$$
\begin{aligned}
& \frac{d S}{d t}=\Lambda-\beta(I) S(t) \int_{0}^{\tau} I(t-s) d \eta(s)-\mu_{1} S(t)+\delta R(t), \\
& \frac{d I}{d t}=\beta(I) S(t) \int_{0}^{\tau} I(t-s) d \eta(s)-\left(\mu_{2}+\gamma\right) I(t), \\
& \frac{d R}{d t}=\gamma I(t)-\left(\mu_{3}+\delta\right) R(t),
\end{aligned}
$$

Here $\delta>0$ is the rate of removed individuals who lose immunity and return to susceptible class. $\gamma>0$ is the recovery rate of infected individuals. All positive constants $\mu_{1}, \mu_{2}$ and $\mu_{3}$ represent the death rates of the susceptible, infected and recovered, respectively. In particular, we consider a variable incidence rate function(infected dependent) $\beta(I)=\beta_{0}+\beta_{1} I(t)\left(\beta_{0}>\right.$ $\left.0, \beta_{1} \geq 0\right)$. The aim of this paper is to prove the existence of solution of the $S I R S$ epidemic model (1.1)-(1.3) and investigate some asymptotic behaviors of the model (1.1)-(1.3). 


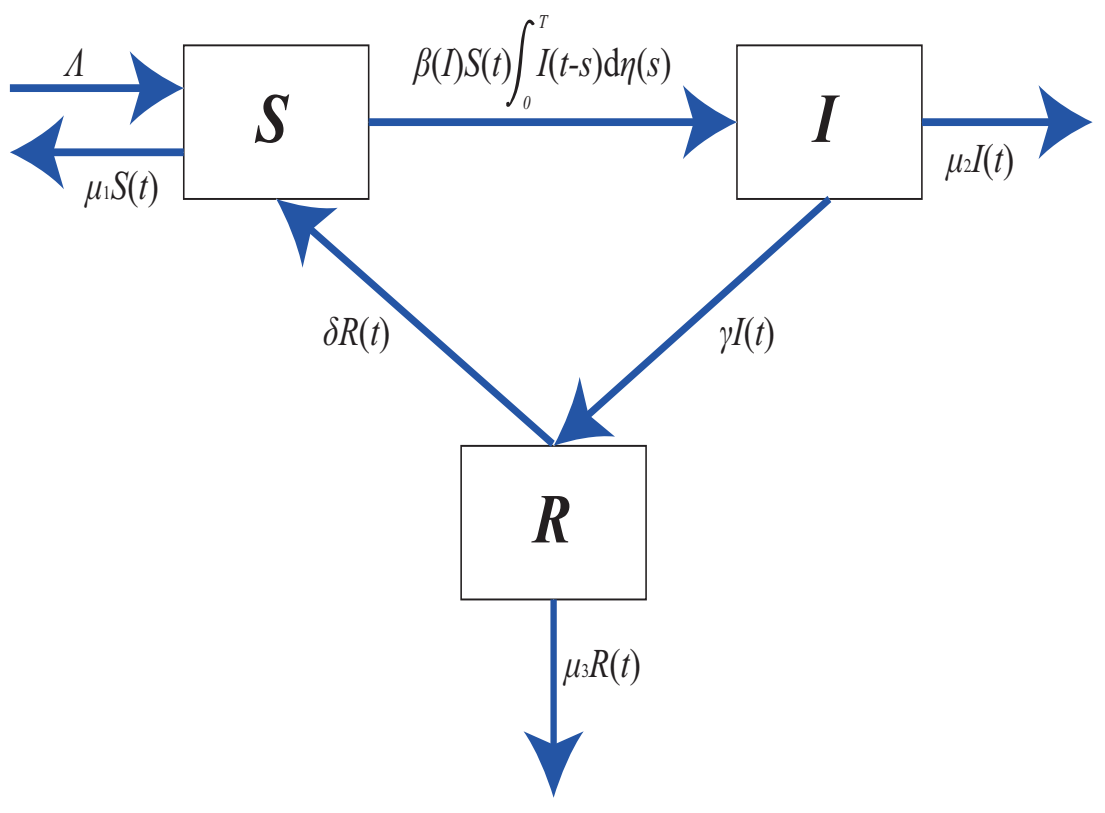

FIGURE 1. Flow chart of the mathematical model for the dynamics of epidemics prevalence in the individual.

We remark that Beretta et al.[2] considered the measure $f(s) d s$ instead of the measure $d \eta(s)$ in the model (1.1)-(1.3). Here, $f(s)$ is the fraction of vector population in which the time taken to become infectious is $s$. That is assumed to be a nonnegative function on $[0, \tau]: f:[0, \tau] \rightarrow$ $[0, \infty)$ square integrable on $[0, \tau]$ and that satisfies:

$$
\int_{0}^{\tau} f(s) d s=1, \int_{0}^{\tau} s f(s) d s<+\infty .
$$

In fact, they studied global stability of an $S I R$ epidemic model with time delays. Zhang and Teng [8] also considered a similar form to the model (1.1)-(1.3), but they used a constant $\beta$ of incidence rate only. They studied global behavior of equilibria and permanence of the disease about an SIRS epidemic model with time delay. We remark also that the nonlinear incidence rates of another forms were studied(see $[9,11,12]$ ).

This paper is organized as follows: In Section 2, we prove the existence of solutions of the model (1.1)-(1.3) and derive the reproduction number and equilibria of the model (1.1)-(1.3). In Section 3, stability analysis for the SIRS epidemic model and an illustrating example is given. 


\section{EXISTENCE, EQUILIBRIA, AND REPRODUCTION NUMBER}

In this section, we will show the existence and uniqueness of solutions for the model (1.1)-(1.3) and find the equilibria and reproduction number. First the initial condition of the model (1.1)-(1.3) is given as

$$
S(\theta)=\varphi_{1}(\theta), I(\theta)=\varphi_{2}(\theta), R(\theta)=\varphi_{3}(\theta),-\tau \leq \theta \leq 0,
$$

where $\Psi=\left(\varphi_{1}, \varphi_{2}, \varphi_{3}\right)^{T} \in C$ such that $\varphi_{i}(\theta) \geq 0(i=1,2,3)$ for all $-\tau \leq \theta \leq 0$, and $\mathcal{C}$ denote the Banach space $\mathcal{C}\left([-\tau, 0], \mathbb{R}^{3}\right)$ of continuous functions mapping interval $[-\tau, 0]$ into $\mathbb{R}^{3}$ and designates the norm of an element $\Psi$ in $\mathcal{C}$ by $\|\Psi\|=\sup _{-\tau \leq \theta \leq 0}\left\{\left|\varphi_{1}(\theta)\right|,\left|\varphi_{2}(\theta)\right|,\left|\varphi_{3}(\theta)\right|\right\}$. By a biological meaning, we further assume that $\varphi_{i}(0)>0$ for $i=1,2,3$.

Let

$$
\begin{gathered}
\Psi=\left[\begin{array}{l}
S(t) \\
I(t) \\
R(t)
\end{array}\right], \quad A=\left[\begin{array}{ccc}
-\mu_{1} & 0 & \delta \\
0 & -\left(\mu_{2}+\gamma\right) & 0 \\
0 & \gamma & -\left(\mu_{3}+\delta\right)
\end{array}\right], \\
B(\Psi)=\left[\begin{array}{c}
\Lambda-\beta\left(\varphi_{2}\right) \varphi_{1}(t) \int_{0}^{\tau} \varphi_{2}(t-s) \eta(s) \\
\beta\left(\varphi_{2}\right) \varphi_{1}(t) \int_{0}^{\tau} \varphi_{2}(t-s) \eta(s) \\
0
\end{array}\right] .
\end{gathered}
$$

Then we rewrite the model (1.1)-(1.3) in the following form:

$$
\frac{d \Psi}{d t}=A \Psi+B(\Psi)
$$

We can see that the second term on the right hand side of Equation (2.2) satisfies

$$
\begin{aligned}
B\left(\Psi_{1}\right)-B\left(\Psi_{2}\right) \leq & \left(\left|\beta \int_{0}^{\tau} I_{1}(t-s) d \eta(s)\right|\right. \\
& \left.+\left|\beta \beta_{0} S_{1}(t) \int_{0}^{\tau} I_{1}(t-s) d \eta(s)\right|\right)\left|I_{1}(t)-I_{2}(t)\right| \\
& +\left|\beta \beta_{0} I_{2}(t) \int_{0}^{\tau} I_{2}(t-s) d \eta(s)\right|\left|S_{1}(t)-S_{2}(t)\right| \\
& +\left(\left|\beta I_{2}\right|+\left|\beta \beta_{0} S_{1}(t) I_{2}(t)\right|\right) A \sup _{-\tau \leq t \leq 0}\left|I_{1}(t-s)-I_{2}(t-s)\right| \\
\leq & M\left(\left|S_{1}(t)-S_{2}(t)\right|+\left|I_{1}(t)-I_{2}(t)\right|\right),
\end{aligned}
$$

where the constant $M>0$ is independent of the state variable $S(t)$ and $I(t)$ less then $\Lambda / \mu$. Let $L=\max \{M,\|A\|\}<\infty$. Thus, we get $\left|B\left(\Psi_{1}\right)-B\left(\Psi_{2}\right)\right| \leq L\left|\Psi_{1}-\Psi_{2}\right|$, it means that the function $B$ is uniformly Lipschitz continuous on $\mathcal{C}$. Therefore, we have the following result:

Theorem 2.1. There exists a unique solution $(S(t), I(t), R(t))$ of the model (1.1)-(1.3) with the initial condition (2.1). 
Now we will find Equilibria and the basic reproduction number. Let $(\bar{S}, \bar{I}, \bar{R})$ be an equilibrium point of the model (1.1)-(1.3). These equilibria can be found by solving the following equations for $\bar{S}, \bar{I}$ and $\bar{R}$ :

$$
\begin{aligned}
& \Lambda-\left(\beta_{0}+\beta_{1} \bar{I}\right) \overline{S I} A-\mu_{1} \bar{S}+\delta \bar{R}=0, \\
& \left(\beta_{0}+\beta_{1} \bar{I}\right) \overline{S I} A-\left(\mu_{2}+\gamma\right) \bar{I}=0, \\
& \gamma \bar{I}-\left(\mu_{3}+\delta\right) \bar{R}=0 .
\end{aligned}
$$

By (2.4), we get $\bar{I}=0$ or $\left(\beta_{0}+\beta_{1} \bar{I}\right) \bar{S} A-\left(\mu_{2}+\gamma\right)=0$. Substituting $\bar{I}=0$ into (2.3) and (2.5) we get $\bar{R}=0$ and $\bar{S}=\Lambda / \mu_{1}$. Thus, disease free equilibrium is $\left(\Lambda / \mu_{1}, 0,0\right)$.

Since the mean infected period is $1 /\left(\mu_{2}+\gamma\right)$ and the rate of secondary infection is $\beta_{0} \bar{S} \int_{0}^{\tau} d \eta(s)$, we can see that the basic reproduction number of the model (1.1)-(1.3) is

$$
\mathcal{R}_{0}=\beta_{0} \Lambda A / \mu_{1}\left(\mu_{2}+\gamma\right) \text {. }
$$

To obtain endemic equilibria of the system(1.1)-(1.3), by solving for $\bar{I}$ when $\bar{I} \neq 0,\left(\beta_{0}+\right.$ $\left.\beta_{1} \bar{I}\right) \bar{S} A-\left(\mu_{2}+\gamma\right)=0$, we must consider the following equation:

$$
a \bar{I}^{2}+b \bar{I}+c=0
$$

where the constants $a, b$ and $c$ are given by

$$
\begin{aligned}
a & =A \beta_{1}\left\{\mu_{2}\left(\mu_{3}+\delta\right)+\mu_{3} \gamma\right\}, \\
b & =A \beta_{0}\left\{\mu_{2}\left(\mu_{3}+\delta\right)+\mu_{3} \gamma\right\}-\beta_{1} A \Lambda\left(\mu_{3}+\delta\right), \\
c & =\mu_{1}\left(\mu_{2}+\gamma\right)\left(\mu_{3}+\delta\right)\left(1-\mathcal{R}_{0}\right) .
\end{aligned}
$$

This equation may admit

$$
\bar{I}_{1}=\frac{-b-\sqrt{b^{2}-4 a c}}{2 a}, \bar{I}_{2}=\frac{-b+\sqrt{b^{2}-4 a c}}{2 a} .
$$

Set $\bar{I}_{1}=\left(-b-\sqrt{b^{2}-4 a c}\right) / 2 a, \bar{I}_{2}=\left(-b+\sqrt{b^{2}-4 a c}\right) / 2 a, \bar{R}_{1}=\gamma \bar{I}_{1} /\left(\mu_{3}+\delta\right), \bar{R}_{2}=$ $\gamma \bar{I}_{2} /\left(\mu_{3}+\delta\right), \bar{I}_{*}=\left(\bar{I}_{1}+\bar{I}_{2}\right) / 2, \bar{R}_{*}=\gamma \bar{I}_{*} /\left(\mu_{3}+\delta\right)$, and

$$
\mathcal{R}_{0}{ }^{*}=1-b^{2} / 4 a \mu_{1}\left(\mu_{2}+\gamma\right)\left(\mu_{3}+\delta\right) .
$$

If $\mathcal{R}_{0}>1$, then $c<0$ and $a$ is always positive. Since $b^{2}-4 a c>0$ and $|b|<\sqrt{b^{2}-4 a c}$, $\bar{I}_{1}$ is negative and $\bar{I}_{2}$ is positive regardless of sign $b$. Thus a positive solution of Equation (2.6) is only $E_{2}=\left(\bar{S}_{2}, \bar{I}_{2}, \bar{R}_{2}\right)$. If $\mathcal{R}_{0}^{*}<\mathcal{R}_{0}<1$, then $c>0$ and $|b|>\sqrt{b^{2}-4 a c} . \bar{I}_{1}$ and $\bar{I}_{2}$ are positive when $-b>0$. Thus positive solutions of Equation (2.6) are $E_{1}=\left(\bar{S}_{1}, \bar{I}_{1}, \bar{R}_{1}\right)$ and $E_{2}=\left(\bar{S}_{2}, \bar{I}_{2}, \bar{R}_{2}\right)$. If $\mathcal{R}_{0}<\mathcal{R}_{0}^{*}$, then $b^{2}-4 a c<0$ and so there is no solution.

Therefore we can state the following results:

Proposition 2.2. (i) The model (1.1)-(1.3) always has a disease free equilibrium.

(ii) The model (1.1)-(1.3) has no endemic equilibrium if $\mathcal{R}_{0}<\mathcal{R}_{0}^{*}$

(iii) The model (1.1)-(1.3) has two endemic equilibrium if $\mathcal{R}_{0}^{*}<\mathcal{R}_{0}<1$ and $b<0$

(iv) The model (1.1)-(1.3) has exactly one endemic equilibrium if $\mathcal{R}_{0}>1$. 


\section{STABILITY ANALYSIS}

In this section, we discuss the asymptotic behavior of the model (1.1)-(1.3). From the biological consideration, we study the model (1.1)-(1.3) in the closed set

$$
\Omega=\left\{(S, I, R) \in \mathbb{R}^{3}: S \geq 0, I \geq 0, R \geq 0, S+I+R \leq \frac{\Lambda}{\mu_{1}}\right\} .
$$

Lemma 3.1. If $(S(\theta), I(\theta), R(\theta)) \in \Omega$ for all $\theta \in[-\tau, 0]$, then $(S(t), I(t), R(t)) \in \Omega$ for all $t \geq 0$.

Proof. See Zhang and Teng [8].

\subsection{Disease free equilibrium}

It may be that the stability varies according to the value of $\mathcal{R}_{0}$. Specifically, when $\mathcal{R}_{0}<1$, it will appear locally asymptotically stable or globally asymptotically stable according to $\mathcal{R}_{0}^{*}$.

Theorem 3.2. For the model (1.1)-(1.3) with initial condition(2.1), if $\mathcal{R}_{0}<1$, then the disease free equilibrium is locally asymptotic stable. But it is unstable if $\mathcal{R}_{0}>1$. Furthermore if $\mathcal{R}_{0}<\mathcal{R}_{0}^{*}<1$, then the equilibrium is globally asymptotically stable.

Proof. Set

$$
x(t)=S(t)-\bar{S}, y(t)=I(t)-\bar{I}, z(t)=R(t)-\bar{R} .
$$

Then it follows from the model (1.1)-(1.3) that

$$
\begin{aligned}
\frac{d x}{d t}= & -\left(\beta_{0}+\beta_{1} y(t)+\beta_{1} \bar{I}\right)(x(t)+\bar{S}) \int_{0}^{\tau} y(t-s) d \eta(s)-\beta_{1} \bar{I} A x(t) y(t) \\
& -\left(\beta_{0} A \bar{I}+\beta_{1} A \bar{I}^{2}+\mu_{1}\right) x(t)-\beta_{1} \overline{S I} A y(t)+\delta z(t), \\
\frac{d y}{d t}= & \left(\beta_{0}+\beta_{1} y(t)+\beta_{1} \bar{I}\right)(x(t)+\bar{S}) \int_{0}^{\tau} y(t-s) d \eta(s) \\
& +\left(\beta_{0}+\beta_{1} \bar{I}\right) \bar{I} A x(t)-\left[\beta_{1} \overline{S I} A-\left(\mu_{2}+\gamma\right)\right] y(t)+\beta_{1} \bar{I} A y(t) x(t), \\
\frac{d z}{d t}= & \gamma y(t)-\left(\mu_{3}+\delta\right) z(t) .
\end{aligned}
$$

The linear part of the system (3.1)-(3.3) is

$$
\begin{aligned}
\frac{d x}{d t}= & -\left(\beta_{0}+\beta_{1} \bar{I}\right) \bar{S} \int_{0}^{\tau} y(t-s) d \eta(s)-\left(\beta_{0} A \bar{I}+\beta_{1} A \bar{I}^{2}+\mu_{1}\right) x(t) \\
& -\beta_{1} \overline{S I} A y(t)+\delta z(t), \\
\frac{d y}{d t}= & \left(\beta_{0}+\beta_{1} \bar{I}\right) \bar{S} \int_{0}^{\tau} y(t-s) d \eta(s)+\left(\beta_{0}+\beta_{1} \bar{I}\right) \bar{I} A x(t) \\
& -\left[\beta_{1} \overline{S I} A-\left(\mu_{2}+\gamma\right)\right] y(t), \\
\frac{d z}{d t}= & \gamma y(t)-\left(\mu_{3}+\delta\right) z(t) .
\end{aligned}
$$


Let a solution of the exponential form to the system (3.4)-(3.6) be as follow:

$$
x(t)=x_{0} e^{\lambda t}, y(t)=y_{0} e^{\lambda t}, z(t)=z_{0} e^{\lambda t} .
$$

Substituting (3.7) into the system (3.4)-(3.6) yield

$$
\lambda\left[\begin{array}{l}
x_{0} \\
y_{0} \\
z_{0}
\end{array}\right]=\left[\begin{array}{ccc}
-\left(\beta(\bar{I}) A \bar{I}-\mu_{1}\right. & -B & \delta \\
\beta(\bar{I}) A \bar{I} & B-\left(\mu_{2}+\gamma\right) & 0 \\
0 & \gamma & -\left(\mu_{3}+\delta\right)
\end{array}\right]\left[\begin{array}{l}
x_{0} \\
y_{0} \\
z_{0}
\end{array}\right],
$$

where $\beta(\bar{I})=\left(\beta_{0}+\beta_{1} \bar{I}\right), B=\beta_{1} \overline{S I}+\beta(\bar{I}) \bar{S} \int_{0}^{\tau} e^{-\lambda s} d \eta(s)$.

The characteristic equation at $E_{0}=\left(\Lambda / \mu_{1}, 0,0\right)$ is

$$
\left(\lambda+\mu_{1}\right)\left(\lambda+\mu_{2}+\gamma-\beta_{0} S_{0} \int_{0}^{\tau} e^{-\lambda s} d \eta(s)\right)\left(\lambda+\mu_{3}+\delta\right)=0 .
$$

Since $\mu_{1}, \mu_{3}+\delta$ are positive, $\lambda_{1}=-\mu_{1}$ and $\lambda_{2}=-\left(\mu_{3}+\delta\right)$ are negative.

Let

$$
f(\lambda)=\lambda+\mu_{2}+\gamma-\beta_{0} S_{0} \int_{0}^{\tau} e^{-\lambda s} d \eta(s) .
$$

If $\mathcal{R}_{0}>1$, then $f(0)=\mu_{2}+\gamma-\beta_{0} S_{0} A<0, f(\lambda) \rightarrow+\infty$ as $\lambda \rightarrow \infty$. Hence there must exist a value $\lambda_{3}>0$ such that $f\left(\lambda_{3}\right)=0$. Thus the characteristic equation (3.8) has at least one solution with positive real part. Therefore, $E_{0}$ is unstable when $\mathcal{R}_{0}>1$. If $\mathcal{R}_{0}<1$, then $f(0)=\mu_{2}+\gamma-\beta_{0} S_{0} A>0, f(\lambda) \rightarrow-\infty$ as $\lambda \rightarrow-\infty$. Thus there must exist a value $\lambda_{3}<0$ such that $f\left(\lambda_{3}\right)=0$. Hence all solutions of the characteristic equation (3.8) have a negative real part. Therefore, $E_{0}$ is locally asymptotically stable when $\mathcal{R}_{0}<1$. Furthermore, the model (1.1)-(1.3) has a unique equilibrium $\left(\Lambda / \mu_{1}, 0,0\right)$ when $\mathcal{R}_{0}<\mathcal{R}_{0}^{*}<1$. Hence all solutions must be asymptotic to $\left(\Lambda / \mu_{1}, 0,0\right)$ and the disease free equilibrium is globally asymptotically stable.

\subsection{Endemic equilibrium}

Set

$$
X=\mu_{1}^{2}+3 \mu_{1} \mu_{3}+\mu_{3}^{2}, Y=\frac{\left(\mu_{1}+\mu_{3}\right)^{2}}{\mu_{3}+\delta}-\mu_{1}\left(\mu_{2}+\mu_{3}\right) .
$$

We note here that $\mu_{i}(i=1,2,3)$, and $\delta$ are parameters defined by the model (1.1)-(1.3).

Theorem 3.3. If the parameters satisfy the following two conditions;

$$
\begin{aligned}
& \beta\left(\bar{I}_{2}\right)^{2}\left\{4 \mu_{1} \mu_{2} X-Y^{2}\right\}+\left(3 X+\mu_{1} \mu_{3}\right) \mu_{1}{ }^{2} \beta_{1}{ }^{2} \bar{S}_{2}{ }^{2} \\
& \quad-2 \beta\left(\bar{I}_{2}\right)\left[\left(\mu_{1}+\mu_{3}\right) Y-2\left(\mu_{1}+\mu_{2}\right) X \bar{S}_{2} \beta_{1} \mu_{1}\right]>0,
\end{aligned}
$$

and

(ii) $\left[3 \mu_{1} \beta_{1} \bar{S}_{1}-2 \beta\left(\bar{I}_{2}\right)\left(\mu_{1}+\mu_{2}\right)\right] X+\mu_{1}\left[\mu_{1} \mu_{3} \beta_{1} \bar{S}_{2}-\beta\left(\bar{I}_{2}\right)\left(\mu_{1}+\mu_{3}\right) Y\right]<0$,

then the endemic equilibrium $E_{2}$ of the model (1.1)-(1.3) is asymptotically stable. 
Proof. Let us define a Lyapunov function $V\left(u_{t}\right)$ for the system (3.4)-(3.6) as follows,

$$
\begin{gathered}
V\left(u_{t}\right)=\frac{1}{2} \omega_{1}(x(t)+y(t)+z(t))^{2}+\frac{1}{2} \omega_{2} y^{2}(t)+\frac{1}{2} \omega_{3} z^{2}(t) \\
+\frac{1}{2} \omega_{2} \beta\left(\bar{I}_{2}\right) \bar{S}_{2} \int_{0}^{\tau} \int_{t-s}^{t} y^{2}(u) d u d \eta(s) .
\end{gathered}
$$

Here, $u_{t}=\left(x_{t}, y_{t}, z_{t}\right)$ and $\omega_{i}>0(i=1,2,3)$ will be given later. Then we obtain the time derivative along the solution of (3.4)-(3.6) as follows:

$$
\begin{aligned}
\frac{d V}{d t} \leq & -\omega_{1} \mu_{1} x^{2}(t)-\left[\omega_{1} \mu_{2}-\omega_{2} \beta_{1} \bar{S}_{2} \bar{I}_{2} A\right] y^{2}(t) \\
& -\left[\omega_{1} \mu_{3}+\omega_{3}\left(\mu_{3}+\delta\right)\right] z^{2}(t)-\omega_{1}\left(\mu_{1}+\mu_{3}\right) x(t) z(t) \\
& +\left[\omega_{3} \gamma-\omega_{1}\left(\mu_{2}+\mu_{3}\right)\right] y(t) z(t)+\left[\omega_{2} \beta\left(\bar{I}_{2}\right) \bar{I}_{2} A-\omega_{1}\left(\mu_{1}+\mu_{2}\right)\right] x(t) y(t) \\
= & \left\{\left(\mu_{1}+\mu_{3}\right)^{2}-4 \mu_{1}\left[\mu_{3}+\omega_{3}\left(\mu_{3}+\delta\right)\right]\right\} z^{2}(t) \\
& +\left\{-2\left[\omega_{2} \beta\left(\bar{I}_{2}\right) \bar{I}_{2} A-\omega_{1}\left(\mu_{1}+\mu_{2}\right)\right]\left(\mu_{1}+\mu_{3}\right)+\left[\omega_{3} \gamma-\left(\mu_{2}+\mu_{3}\right)\right] 4 \mu_{1}\right\} \\
& \times z(t) y(t)+\left\{\left[\omega_{2} \beta\left(\bar{I}_{2}\right) \bar{I}_{2} A-\left(\mu_{1}+\mu_{2}\right)\right]^{2}-4 \mu_{1}\left(\mu_{2}-\omega_{2} \beta_{1} \bar{S}_{2} \bar{I}_{2} A\right)\right\} y^{2}(t) .
\end{aligned}
$$

Let

$$
\frac{d V}{d t} \leq p z^{2}(t)+q z(t) y(t)+m y^{2}(t)
$$

where

$$
\begin{aligned}
& p=\left\{\left(\mu_{1}+\mu_{3}\right)^{2}-4 \mu_{1}\left[\mu_{3}+\omega_{3}\left(\mu_{3}+\delta\right)\right]\right\}, \\
& q=\left\{-2\left[\omega_{2} \beta\left(\bar{I}_{2}\right) \bar{I}_{2} A-\omega_{1}\left(\mu_{1}+\mu_{2}\right)\right]\left(\mu_{1}+\mu_{3}\right)+\left[\omega_{3} \gamma-\left(\mu_{2}+\mu_{3}\right)\right] 4 \mu_{1}\right\}, \\
& m=\left\{\left[\omega_{2} \beta\left(\bar{I}_{2}\right) \bar{I}_{2} A-\left(\mu_{1}+\mu_{2}\right)\right]^{2}-4 \mu_{1}\left(\mu_{2}-\omega_{2} \beta_{1} \bar{S}_{2} \bar{I}_{2} A\right)\right\} .
\end{aligned}
$$

So $d V / d t$ is negative definite if and only if (i) $p<0$, (ii) $q^{2}-4 p m<0$.

Let us choose $\omega_{3}$ satisfying $p<0$. Then we get

$$
\begin{gathered}
\left\{\left(\mu_{1}+\mu_{3}\right)^{2}-4 \mu_{1}\left[\mu_{3}+\omega_{3}\left(\mu_{3}+\delta\right)\right]\right\}<0 \\
\frac{\left(\mu_{1}-\mu_{3}\right)^{2}}{4 \mu_{1}\left(\mu_{3}+\delta\right)}<\omega_{3} .
\end{gathered}
$$


So we can choose $\omega_{3}=\left(\mu_{1}+\mu_{3}\right)^{2} / \mu_{1}\left(\mu_{3}+\delta\right)>0$. If we choose $\omega_{1}=1$, then we obtain

$$
\begin{aligned}
q^{2}-4 p m= & \left\{-2\left[\omega_{2} \beta\left(I_{1}\right) I_{1} A-\left(\mu_{1}+\mu_{2}\right)\right]\left(\mu_{1}+\mu_{3}\right)+\left[\omega_{3} \gamma-\left(\mu_{2}+\mu_{3}\right)\right] 4 \mu_{1}\right\}^{2} \\
& -4\left\{\left(\mu_{1}+\mu_{3}\right)^{2}-4 \mu_{1}\left[\mu_{3}+\omega_{3}\left(\mu_{3}+\delta\right)\right]\right\}\left\{\left[\omega_{2} \beta\left(\bar{I}_{2}\right) \bar{I}_{2} A-\left(\mu_{1}+\mu_{2}\right)\right]^{2}\right. \\
& \left.-4 \mu_{1}\left(\mu_{2}-\omega_{2} \beta_{1} \bar{S}_{2} \bar{I}_{2} A\right)\right\} \\
= & 4\left(\mu_{1}^{2}+3 \mu_{1} \mu_{3}+\mu_{3}^{2}\right) \beta\left(\bar{I}_{2}\right)^{2} \bar{I}_{2}{ }^{2} A^{2} \omega_{2}^{2}+\left\{-8\left(\mu_{1}{ }^{2}+3 \mu_{1} \mu_{3}+\mu_{3}^{2}\right)\right. \\
& \times \beta\left(\bar{I}_{2}\right) \bar{I}_{2} A^{2}\left(\mu_{1}+\mu_{2}\right)-4 \mu_{1} \beta\left(\bar{I}_{2}\right) \bar{I}_{2} A\left(\mu_{1}+\mu_{3}\right)\left[\frac{\left(\mu_{1}+\mu_{3}\right)^{2}}{\mu_{3}+\delta}\right. \\
& \left.\left.-\mu_{1}\left(\mu_{2}+\mu_{3}\right)\right]-4 \mu_{1} \beta_{1} \bar{S}_{2} \bar{I}_{2} A\left[\left(\mu_{1}-\mu_{3}\right)^{2}-4\left(\mu_{1}+\mu_{3}\right)^{2}\right]\right\} \omega_{2} \\
& +\left\{4\left(\mu_{1}+\mu_{2}\right)^{2}\left(\mu_{1}{ }^{2}+3 \mu_{1} \mu_{3}+\mu_{3}{ }^{2}\right)+4 \mu_{1}\left(\mu_{1}+\mu_{2}\right)\left(\mu_{1}+\mu_{3}\right)\right. \\
& \times\left[\frac{\left(\mu_{1}+\mu_{3}\right)^{2}}{\mu_{3}+\delta}-\mu_{1}\left(\mu_{2}+\mu_{3}\right)\right]+4 \mu_{1}{ }^{2}\left[\frac{\left(\mu_{1}+\mu_{3}\right)^{2}}{\mu_{3}+\delta}-\mu_{1}\left(\mu_{2}+\mu_{3}\right)\right]^{2} \\
& \left.+4 \mu_{1} \mu_{2}\left[\left(\mu_{1}-\mu_{3}\right)^{2}-4\left(\mu_{1}+\mu_{3}\right)^{2}\right]\right\} .
\end{aligned}
$$

Set

$$
\begin{aligned}
e= & 4\left(\mu_{1}^{2}+3 \mu_{1} \mu_{3}+\mu_{3}^{2}\right) \beta\left(\bar{I}_{2}\right)^{2} \bar{I}_{2}^{2} A^{2} \\
f= & -8\left(\mu_{1}^{2}+3 \mu_{1} \mu_{3}+\mu_{3}^{2}\right) \beta\left(\bar{I}_{2}\right) \bar{I}_{2} A^{2}\left(\mu_{1}+\mu_{2}\right)-4 \mu_{1} \beta\left(\bar{I}_{2}\right) \bar{I}_{2} A\left(\mu_{1}+\mu_{3}\right) \\
& \times\left[\frac{\left(\mu_{1}+\mu_{3}\right)^{2}}{\mu_{3}+\delta}-\mu_{1}\left(\mu_{2}+\mu_{3}\right)\right]-4 \mu_{1} \beta_{1} \bar{S}_{2} \bar{I}_{2} A\left[\left(\mu_{1}-\mu_{3}\right)^{2}-4\left(\mu_{1}+\mu_{3}\right)^{2}\right] \\
g= & 4\left(\mu_{1}+\mu_{2}\right)^{2}\left(\mu_{1}^{2}+3 \mu_{1} \mu_{3}+\mu_{3}^{2}\right)+4 \mu_{1}\left(\mu_{1}+\mu_{2}\right)\left(\mu_{1}+\mu_{3}\right) \\
& \times\left[\frac{\left(\mu_{1}+\mu_{3}\right)^{2}}{\mu_{3}+\delta}-\mu_{1}\left(\mu_{2}+\mu_{3}\right)\right]+4 \mu_{1}{ }^{2}\left[\frac{\left(\mu_{1}+\mu_{3}\right)^{2}}{\mu_{3}+\delta}-\mu_{1}\left(\mu_{2}+\mu_{3}\right)\right]^{2} \\
& +4 \mu_{1} \mu_{2}\left[\left(\mu_{1}-\mu_{3}\right)^{2}-4\left(\mu_{1}+\mu_{3}\right)^{2}\right] .
\end{aligned}
$$

Denote $h=\omega_{2}, H(h)=e h^{2}+f h+g$. Then we obtain by condition $(i)$,

$$
\begin{aligned}
f^{2}-4 e g= & 16 \bar{I}_{2}{ }^{2} A^{2}\left(3 \mu_{1}{ }^{2}+10 \mu_{1} \mu_{2}+3 \mu_{3}{ }^{2}\right)\left\{\beta ( \overline { I } _ { 2 } ) ^ { 2 } \left\{4 \mu_{1} \mu_{2}\left(\mu_{1}{ }^{2}+3 \mu_{1} \mu_{3}+\mu_{3}{ }^{2}\right)\right.\right. \\
& \left.-\left[\frac{\left(\mu_{1}+\mu_{3}\right)^{2}}{\mu_{3}+\delta}-\mu_{1}\left(\mu_{2}+\mu_{3}\right)\right]^{2}\right\}+\bar{S}_{2} \beta_{1} \mu_{1}\left\{\overline { S } _ { 2 } \beta _ { 1 } \mu _ { 1 } \left(3 \mu_{1}{ }^{2}\right.\right. \\
& \left.+10 \mu_{1} \mu_{2}+3 \mu_{3}{ }^{2}\right)-2 \beta\left(\bar{I}_{2}\right)\left(\mu_{1}+\mu_{3}\right)\left[\frac{\left(\mu_{1}+\mu_{3}\right)^{2}}{\mu_{3}+\delta}-\mu_{1}\left(\mu_{2}+\mu_{3}\right)\right] \\
& \left.-4 \beta\left(\bar{I}_{2}\right)\left(\mu_{1}+\mu_{2}\right)\left(\mu_{1}{ }^{2}+3 \mu_{1} \mu_{3}+\mu_{3}{ }^{2}\right)\right\} \\
= & 16 \bar{I}_{2}{ }^{2} A^{2}\left(3 \mu_{1}{ }^{2}+10 \mu_{1} \mu_{2}+3 \mu_{3}{ }^{2}\right)\left\{\beta\left(\bar{I}_{2}\right)^{2}\left\{4 \mu_{1} \mu_{2} X-Y^{2}\right\}\right. \\
& +\left(3 X+\mu_{1} \mu_{3}\right) \mu_{1}{ }^{2} \beta_{1}{ }^{2} \bar{S}_{2}{ }^{2}-2 \beta\left(\bar{I}_{2}\right)\left[\left(\mu_{1}+\mu_{3}\right) Y\right. \\
& \left.\left.-2\left(\mu_{1}+\mu_{2}\right) X \bar{S}_{2} \beta_{1} \mu_{1}\right]\right\}>0 .
\end{aligned}
$$


Since $\mu_{i}>0(i=1,2,3), \bar{I}_{2}>0$ and $A>0$, the coefficient $e$ is positive. From condition (ii) we obtain

$$
\begin{aligned}
f= & \left\{-8\left(\mu_{1}^{2}+3 \mu_{1} \mu_{3}+\mu_{3}^{2}\right) \beta\left(\bar{I}_{2}\right) \bar{I}_{2} A\left(\mu_{1}+\mu_{2}\right)-4 \mu_{1} \beta\left(\bar{I}_{2}\right) \bar{I}_{2} A\left(\mu_{1}+\mu_{3}\right)\right. \\
& \left.\times\left[\frac{\left(\mu_{1}+\mu_{3}\right)^{2}}{\mu_{3}+\delta}-\mu_{1}\left(\mu_{2}+\mu_{3}\right)\right]-4 \mu_{1} \beta_{1} \bar{S}_{2} \bar{I}_{2} A\left[\left(\mu_{1}-\mu_{3}\right)^{2}-4\left(\mu_{1}+\mu_{3}\right)^{2}\right]\right\} \\
= & {\left[3 \mu_{1} \beta_{1} \bar{S}_{2}-2 \beta\left(\bar{I}_{2}\right)\left(\mu_{1}+\mu_{2}\right)\right] X+\mu_{1}\left[\mu_{1} \mu_{3} \beta_{1} \bar{S}_{2}-\beta\left(\bar{I}_{2}\right)\left(\mu_{1}+\mu_{3}\right) Y\right]<0 }
\end{aligned}
$$

so that $f^{2}-4 e g>0$ and $-f / e>0$. Thus we can choose positive real value $\omega_{2}$ and $H\left(\omega_{2}\right)<0$. Choose $\omega_{2}=-f / 2 e$. Then $H\left(\omega_{2}\right)=e \omega_{2}^{2}+f \omega_{2}+g<0$. Therefore $p^{2}-4 q m<0$, that is, $V\left(u_{t}\right)$ is negative definite. We show that endemic equilibrium $E_{2}$ of the model (1.1)-(1.3) is asymptotically stable.

Example 3.4. Let $\Lambda=1, \mu_{1}=0.1, \mu_{2}=0.4, \mu_{3}=0.4, \delta=0.6, \gamma=0.5, A=1$, $\beta_{0}=0.4, \beta_{1}=0.01$ in the model (1.1)-(1.3). Then we have $\mathcal{R}_{0}=4.4444>1, \bar{S}_{2}=2.1790$, $\bar{I}_{2}=1.3035, \bar{R}_{2}=0.6518$ and so we can verify that the parameter conditions in Theorem 3.3 are satisfied; $(i)=0.0023>0,(i i)=-0.1178<0$. Hence, from Theorem 3.3, the endemic equilibrium $E_{2}=(2.1790,1.3035,0.6518)$ of the model (1.1)-(1.3) is asymptotically stable(see Figure 2).

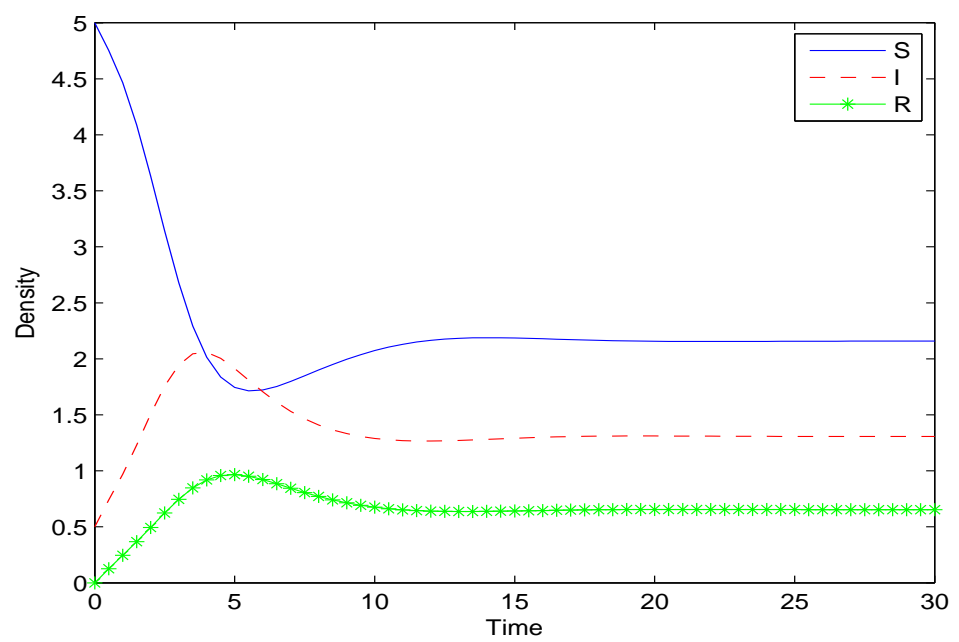

FIGURE 2. Asymptotic Behavior when the parameters $\Lambda=1, \mu_{1}=0.1$, $\mu_{2}=0.4, \mu_{3}=0.4, \delta=0.6, \gamma=0.5, A=1, \beta_{0}=0.4, \beta_{1}=0.01$, $\mathcal{R}_{0}=4.4444$, and the initial condition $\left(S_{0}, I_{0} \cdot R_{0}\right)=(5,0.5,0)$. 


\section{REFERENCES}

[1] N. T. J. Bailey, The Mathematical Theory of Infectious Diseases, 2nd ed., Hafner, New York, 1975.

[2] E. Beretta, T. Hara, W. Ma, and Y. Takeuchi, Convergence results in SIR epidemic models with varying population sizes, J. Appl. Nonlinear Anal. 28 (1997), 1909-1921.

[3] F. Brauer and C. Castillo-Charvez, Mathematical Models in Population Biology and Epidemiology, SpringerVerlag, New York, 2001.

[4] W. H. Herbert, The mathematics of infectious diseases, SIAM Rev. 42 (2000), 599-653.

[5] W. O. Kermack and A. G. Mckendrick, Contribution to the mathematical theory of epidemics, Proc. Roy. Soc. London Ser. A 115, (1927), 700-721.

[6] W. Ma, M. Song, and Y. Takeuchi, Global stability of an SIR epidemic model with time delay, Appl. Math. Lett. 17 (2004), 1141-1145.

[7] G. Schneckenreither, N. Popper, G. Zauner, and F. Breitenecker, Modelling SIR-type epidemics by ODEs, PDEs, difference equations and cellular automata - A comparative study, Simulation Modelling Practice and Theory, 16 (2008), 1014-1023.

[8] Z. Teng and Z. Zhang, Global behavior and permanence of an SIRS epidemic model with time delays, J. Nonlinear Anal. 9 (2008), 1409-1424.

[9] S. Ruan and W. Wang, Dynamical behavior of an epidemic model with a nonlinear incidence rate, J. Differential Equations 188 (2003), 135-163.

[10] G. Zaman, K. H. Kang, and I. H. Jung, Stability analysis and optimal vaccination of an SIR epidemic model, BioSystem 93 (2008), 240-249.

[11] Y. Jin, W. Wang, and S. Xiao, An SIRS model with a nonlinear incidence rate, J. Chaos, Solitons and Fractals 34 (2007), 1482-1497.

[12] P. van den Driessche and J. Watmough, A simple SIS epidemic model with a backward bifurcation, J. Math. Biol. 40 (2000), 525-540. 